\title{
Convergence theorems for modified generalized $f$-projections and generalized nonexpansive mappings
}

\author{
Qingqing Cheng, Yongfu Su* and Jingling Zhang
}

\section{"Correspondence:}

suyongfu@gmail.com

Department of Mathematics, Tianjin

Polytechnic University, Tianjin,

300160, China

\begin{abstract}
The purpose of this paper is to study a sequence of modified generalized $f$-projections in a reflexive, smooth, and strictly convex Banach space and show that Mosco convergence of their ranges implies their pointwise convergence to the generalized $f$-projection onto the limit set. Furthermore, we prove a strong convergence theorem for a countable family of $\alpha$-nonexpansive mappings in a uniformly convex and smooth Banach space using the properties of a modified generalized $f$-projection operator. Our main results generalize the results of Ziming Wang, Yongfu Su, and Jinlong Kang and enrich the research contents of $\alpha$-nonexpansive mappings.
\end{abstract}

MSC: $47 \mathrm{H} 05 ; 47 \mathrm{H} 09 ; 47 \mathrm{H} 10$

Keywords: $\alpha$-nonexpansive mappings; monotone hybrid algorithm; modified generalized $f$-projection operator; Mosco convergence; common fixed point

\section{Introduction}

Let $E$ be a real Banach space and $C$ be a nonempty closed convex subset of $E$. A mapping $T: C \rightarrow C$ is said to be nonexpansive if

$$
\|T x-T y\| \leq\|x-y\|, \quad \forall x, y \in C .
$$

Lots of iterative schemes for nonexpansive mappings have been introduced (see [1-3]); furthermore, many strong convergence theorems for nonexpansive mappings have been proved. On the other hand, there are many nonlinear mappings which are more general than the nonexpansive mapping. Compared to the existing problem of a fixed point of those mappings, the iterative methods for finding a fixed point are also very useful in studying the fixed point theory and the theory of equations in other fields.

In 2007, Gobel and Pineda [4] introduced and studied a new mapping, called $\alpha$-nonexpansive mapping. The mapping is more general than the nonexpansive mapping.

Definition 1.1 For a given multi-index, $\alpha=\left(\alpha_{1}, \alpha_{2}, \ldots, \alpha_{n}\right)$ satisfies $\alpha_{i} \geq 0, i=1,2, \ldots, n$ and $\sum_{i=1}^{n} \alpha_{i}=1$. A mapping $T: C \rightarrow C$ is said to be $\alpha$-nonexpansive if

$$
\sum_{i=1}^{n} \alpha_{i}\left\|T^{i} x-T^{i} y\right\| \leq\|x-y\|, \quad \forall x, y \in C .
$$


In order to show that the class of $\alpha$-nonexpansive mappings is more general than the one of nonexpansive mappings, we give an example [4].

Example 1.2 Let $E=R^{1}$, and

$$
T(x)= \begin{cases}0 & \text { if } x=0 \\ \frac{1}{x} & \text { if } x \in(0,+\infty) .\end{cases}
$$

Then $T$ is not nonexpansive but $\alpha$-nonexpansive.

Proof Obviously, $T$ is not nonexpansive. Taking $x=\frac{1}{2}, y=0$, by the definition of $T x$, we have

$$
\|T x-T y\|=|2-0|>\left|\frac{1}{2}-0\right|=\|x-y\| .
$$

On the other hand, for every $x, y \in[0,+\infty)$, we have

$$
\left\|T^{2} x-T^{2} y\right\|=\|x-y\| .
$$

Therefore, we can affirm that

$$
0\|T x-T y\|+\left\|T^{2} x-T^{2} y\right\|=\|x-y\|
$$

where $\alpha=\left(\alpha_{1}, \alpha_{2}\right)=(0,1)$. Then $T$ is an $\alpha$-nonexpansive mapping but not a nonexpansive one.

If $T$ is a nonexpansive self-mapping, we can imply that $T$ must be an $\alpha$-nonexpansive one, where $\alpha=\left(\alpha_{1}, \alpha_{2}, \ldots, \alpha_{n}\right)=\left(\frac{1}{n}, \ldots, \frac{1}{n}\right)$.

For technical reasons, we always assume that the first coefficient $\alpha_{1}$ is nonzero, that is, $\alpha_{1}>0$. In this case the mapping $T$ satisfies the Lipschitz condition

$$
\|T x-T y\| \leq \frac{1}{\alpha_{1}}\|x-y\|, \quad \forall x, y \in C .
$$

For the $\alpha$-nonexpansive mapping $T, \alpha=\left(\alpha_{1}, \alpha_{2}, \alpha_{3}, \ldots \alpha_{n}\right)$, it is obvious that the mapping

$$
T_{\alpha} x=\sum_{i=1}^{n} \alpha_{i} T^{i} x, \quad \forall x \in C
$$

is nonexpansive. However, the nonexpansiveness of $T_{\alpha}$ is much weaker than (1.2), for instance, it does not entail the continuity of $T$ (see [4]).

In 2010, Klin-eam and Suantai [5] introduced the relation of fixed point sets between an $\alpha$-nonexpansive operator and a $T_{\alpha}$ operator. They gave the following theorem.

Theorem 1.3 (see Theorem 3.1 of Klin-eam and Suantai [5]) Let $C$ be a closed convex subset of a Banach space $E$ and for all $n \in N$, let $\alpha=\left(\alpha_{1}, \alpha_{2}, \ldots, \alpha_{n}\right)$ such that $\alpha_{i} \geq 0, i=$ $1,2, \ldots, n, \alpha_{1}>0$, and $\sum_{i=1}^{n} \alpha_{i}=1$. Let $T$ be an $\alpha$-nonexpansive mapping from $C$ into itself. If $\alpha_{1}>\frac{1}{\sqrt[n-1]{2}}$, then $F(T)=F\left(T_{\alpha}\right)$, where $F(T)$ is the fixed point set of $T$. 
At the same time, they have succeeded in proving the demiclosedness principle for the $\alpha$-nonexpansive mappings.

Theorem 1.4 (see Theorem 3.4 of Klin-eam and Suantai [5]) Let $C$ be a closed convex subset of a Banach space $E$ and for all $n \in N$, let $\alpha=\left(\alpha_{1}, \alpha_{2}, \ldots, \alpha_{n}\right)$ such that $\alpha_{i} \geq 0, i=$ $1,2, \ldots, n, \alpha_{1}>0$, and $\sum_{i=1}^{n} \alpha_{i}=1$. Let $T$ be an $\alpha$-nonexpansive mapping from $C$ into itself. If $\alpha_{1}>\frac{1}{\sqrt[n-1]{2}}$, if $\left\{x_{n}\right\} \subset C$ converges weakly to $x$ and $\left\{x_{n}-T x_{n}\right\}$ converges strongly to 0 as $n \rightarrow \infty$, then $x \in F(T)$.

Recently, Wang et al. [6] proposed the following hybrid algorithm for an $\alpha$-nonexpansive mapping in a Banach space:

$$
\left\{\begin{array}{l}
y_{n}=\left(1-\beta_{n}\right) x_{n}+\beta_{n} T x_{n}, \\
C_{n+1}=\left\{z \in C_{n}:\left\|y_{n}-z\right\| \leq\left\|x_{n}-z\right\|\right\}, \\
x_{n+1}=\Pi_{C_{n+1}} x_{0}, \quad n \in N .
\end{array}\right.
$$

As we know that if $C$ is a nonempty closed convex subset of a Hilbert space $H$ and recall that the (nearest point) projection $P_{C}$ from $H$ onto $C$ assigns to each $x \in H$, and the unique point $P_{C} x \in C$ satisfies the property $\left\|x-P_{C} x\right\|=\min _{y \in C}\|x-y\|$, it is well known that $P_{C}$ is nonexpansive. This fact actually characterizes Hilbert spaces and, consequently, it is not available in more general Banach spaces. We consider the functional defined by

$$
\phi(y, x)=\|y\|^{2}-2\langle y, J x\rangle+\|x\|^{2}, \quad \forall x, y \in E,
$$

where $J$ is the normalized duality mapping and the Banach space is smooth. In this connection, Alber [7] introduced a generalized projection $\Pi_{C}$ from $E$ to $C$ as follows:

$$
\Pi_{C}(x)=\arg \min _{y \in C} \phi(y, x), \quad \forall x \in E
$$

It is obvious from the definition of functional $\phi$ that

$$
(\|y\|-\|x\|)^{2} \leq \phi(y, x) \leq(\|y\|+\|x\|)^{2}, \quad \forall x, y \in E .
$$

If $E$ is a Hilbert space, then $\phi(y, x)=\|y-x\|^{2}$ and $\Pi_{C}$ becomes the metric projection of $E$ onto $C$. The generalized projection $\Pi_{C}: E \rightarrow C$ is a map that assigns to an arbitrary point $x \in E$ the minimum point of the functional $\phi(y, x)$, that is, $\Pi_{C} x=\bar{x}$, where $\bar{x}$ is the solution to the minimization problem

$$
\phi(\bar{x}, x)=\inf _{y \in C} \phi(y, x)
$$

The existence and uniqueness of the operator $\Pi_{C}$ follow from the properties of the functional $\phi(y, x)$ and strict monotonicity of the normalized duality mapping $J$ [8]. It is well known that the metric projection operator plays an important role in nonlinear functional analysis, optimization theory, fixed point theory, nonlinear programming, game theory, variational inequality, and complementarity problems, etc. [8, 9]. In 1994, Alber [7] introduced and studied the generalized projections from Hilbert spaces to uniformly 
convex and uniformly smooth Banach spaces. Moreover, Alber [8] presented some applications of the generalized projections to approximately solve variational inequalities and von Neumann intersection problem in Banach spaces. In 2005, Li [9] extended the generalized projection operator from uniformly convex and uniformly smooth Banach spaces to reflexive Banach spaces and studied some properties of the generalized projection operator with applications to solve the variational inequality in Banach spaces. Later, Wu and Huang [10] introduced a new generalized $f$-projection operator in Banach spaces. They extended the definition of generalized projection operators introduced by Abler [7] and proved some properties of the generalized $f$-projection operator. In 2009, Fan et al. [11] presented some basic results for the generalized $f$-projection operator and discussed the existence of solutions and approximation of the solutions for generalized variational inequalities in noncompact subsets of Banach spaces.

The purpose of this paper is to study a sequence of modified generalized $f$-projections in a reflexive, smooth, and strictly convex Banach space and show that Mosco convergence of their ranges implies their pointwise convergence to the generalized $f$-projection onto the limit set. Furthermore, we prove strong convergence theorem for a countable family of $\alpha$ nonexpansive mappings in a uniformly convex and smooth Banach space using the properties of a modified generalized $f$-projection operator. Our main results generalize the results of Wang et al. [6] and enrich the research contents of $\alpha$-nonexpansive mappings.

\section{Preliminaries}

A Banach space $E$ is said to be strictly convex if $\frac{\|x+y\|}{2}<1$ for $x, y \in E$ with $\|x\|=\|y\|=1$ and $x \neq y$. It is said to be uniformly convex if for each $\epsilon>0$ there is $\delta>0$ such that for $x, y \in E$ with $\|x\|,\|y\| \leq 1$ and $\|x-y\| \geq \epsilon,\|x+y\| \leq 2(1-\delta)$ holds. The space $E$ is said to be smooth if the limit

$$
\lim _{t \rightarrow 0} \frac{\|x+t y\|-\|x\|}{t}
$$

exists for all $x, y \in S(E)=\{x \in E:\|x\|=1\}$. And $E$ is said to be uniformly smooth if the limit (2.1) exists uniformly for all $x, y \in S(E)$.

Remark 2.1 The following basic properties of a Banach space $E$ can be found in Cioranescu [12]:

(i) if $E$ is uniformly convex, then $E$ is reflexive and strictly convex;

(ii) a Banach space $E$ is uniformly smooth if and only if $E^{*}$ is uniformly convex;

(iii) each uniformly convex Banach space $E$ has the Kadec-Klee property, i.e., for any sequence $\left\{x_{n}\right\} \subset E$, if $x_{n} \rightarrow x \in E$ and $\left\|x_{n}\right\| \rightarrow\|x\|$, then $x_{n} \rightarrow x$.

Let $E$ be a real Banach space with the dual $E^{*}$. We denote by $J$ the normalized duality mapping from $E$ to $2^{E^{*}}$ defined by

$$
J x=\left\{f \in E^{*}:\langle x, f\rangle=\|x\|^{2}=\|f\|^{2}\right\}, \quad x \in E .
$$

Many properties of the normalized duality mapping $J$ can be found in Takahashi [13] or Vainberg [14]. We list some properties below for easy reference:

(i) $J$ is a monotone and bounded operator in arbitrary Banach spaces;

(ii) $J$ is a strictly monotone operator in strictly convex Banach spaces; 
(iii) $J$ is a continuous operator in smooth Banach spaces;

(iv) $J$ is a uniformly continuous operator on each bounded set in uniformly smooth Banach spaces;

(v) $J$ is a bijection in smooth, reflexive, and strictly convex Banach spaces;

(vi) $J$ is the identity operator in Hilbert spaces.

Next, we recall the concept of generalized $f$-projector operator, together with its properties. Let $G: C \times E^{*} \rightarrow R \cup\{+\infty\}$ be a functional defined as follows:

$$
G(\xi, \varphi)=\|\xi\|^{2}-2\langle\xi, \varphi\rangle+\|\varphi\|^{2}+2 \rho f(\xi)
$$

where $\xi \in C, \varphi \in E^{*}, \rho$ is a positive number and $f: C \rightarrow R \cup\{+\infty\}$ is proper, convex, and lower semi-continuous. From the definitions of $G$ and $f$, it is easy to see the following properties:

(i) $G(\xi, \varphi)$ is convex and continuous with respect to $\varphi$ when $\xi$ is fixed;

(ii) $G(\xi, \varphi)$ is convex and lower semi-continuous with respect to $\xi$ when $\varphi$ is fixed.

Definition 2.2 ([10]) Let $E$ be a real Banach space with its dual $E^{*}$. Let $C$ be a nonempty, closed, and convex subset of $E$. We say that $\Pi_{C}^{f}: E^{*} \rightarrow 2^{C}$ is a generalized $f$-projection operator if

$$
\Pi_{C}^{f} \varphi=\left\{u \in C: G(u, \varphi)=\inf _{\xi \in C} G(\xi, \varphi)\right\}, \quad \forall \varphi \in E^{*} .
$$

For the generalized $f$-projection operator, $\mathrm{Wu}$ and Huang [10] proved the following basic properties.

Lemma 2.3 ([10]) Let E be a real reflexive Banach space with its dual $E^{*}$, and let $C$ be a nonempty, closed, and convex subset of $E$. Then the following statements hold:

(i) $\Pi_{C}^{f} \varphi$ is a nonempty closed convex subset of $C$ for all $\varphi \in E^{*}$.

(ii) If $E$ is smooth, then for all $\varphi \in E^{*}, x \in \Pi_{C}^{f} \varphi$ if and only if

$$
\langle x-y, \varphi-J x\rangle+\rho f(y)-\rho f(x) \geq 0, \quad \forall y \in C .
$$

(iii) If $E$ is strictly convex and $f: C \rightarrow R \cup\{+\infty\}$ is positive homogeneous (i.e., $f(t x)=t f(x)$ for all $t>0$ such that $t x \in C$, where $x \in C)$, then $\Pi_{C}^{f}$ is a single-valued mapping.

Fan et al. [11] showed that the condition $f$ is positive homogeneous, which appeared in Lemma 2.3, can be removed.

Lemma 2.4 ([11]) Let $E$ be a real reflexive Banach space with its dual $E^{*}$, and let $C$ be a nonempty, closed, and convex subset of $E$. Then if $E$ is strictly convex, then $\Pi_{C}^{f}$ is a singlevalued mapping.

Recall that $J$ is a single-valued mapping when $E$ is a smooth Banach space. There exists a unique element $\varphi \in E^{*}$ such that $\varphi=J x$ for each $x \in E$. This substitution in (2.2) gives

$$
G(\xi, J x)=\|\xi\|^{2}-2\langle\xi, J x\rangle+\|x\|^{2}+2 \rho f(\xi) .
$$

Now, we consider the second generalized $f$-projection operator in a Banach space. 
Definition 2.5 Let $E$ be a real Banach space and $C$ be a nonempty, closed, and convex subset of $E$. We say that $\Pi_{C}^{f}: E \rightarrow 2^{C}$ is a generalized $f$-projection operator if

$$
\Pi_{C}^{f}(x)=\left\{u \in C: G(u, J x)=\inf _{\xi \in C} G(\xi, J x)\right\}, \quad \forall x \in E
$$

We know that the following lemmas hold for the operator $\Pi_{C}^{f}$.

Lemma 2.6 ([15]) Let C be a nonempty, closed, and convex subset of a smooth and reflexive Banach space E. Then the following statements hold:

(i) $\Pi_{C}^{f} x$ is a nonempty closed and convex subset of $C$ for all $x \in E$.

(ii) For all $x \in E, \hat{x} \in \Pi_{C}^{f} x$ if and only if

$$
\langle\hat{x}-y, J x-J \hat{x}\rangle+\rho f(y)-\rho f(x) \geq 0, \quad \forall y \in C .
$$

(iii) If $E$ is strictly convex, then $\Pi_{C}^{f}$ is a single-valued mapping.

Now, we introduce a modified generalized $f$-projection operator. Let $G: C \times E^{*} \rightarrow R$ be a functional defined as follows:

$$
G(\xi, \varphi)=\|\xi\|^{2}-2\langle\xi, \varphi\rangle+\|\varphi\|^{2}+2 \rho f(\xi),
$$

where $\xi \in C, \varphi \in E^{*}, \rho$ is a positive number and $f: C \rightarrow R$ is convex and weakly continuous. From the definitions of $G$ and $f$, it is easy to see the following properties:

(i) $G(\xi, \varphi)$ is convex and continuous with respect to $\varphi$ when $\xi$ is fixed;

(ii) $G(\xi, \varphi)$ is convex and weakly lower semi-continuous with respect to $\xi$ when $\varphi$ is fixed.

Obviously, the other definitions and lemmas hold respectively.

Next, we give the following example [16] which shows that metric projection, generalized projection and generalized $f$-projection are different.

Example 2.7 Let $X=R^{3}$ be provided with the norm

$$
\left\|\left(x_{1}, x_{2}, x_{3}\right)\right\|=\sqrt{\left(x_{1}^{2}+x_{2}^{2}\right)}+\sqrt{\left(x_{2}^{2}+x_{3}^{2}\right)} .
$$

This is a smooth strictly convex Banach space and $C=\left\{x \in R^{3} \mid x_{2}=0, x_{3}=0\right\}$ is a closed and convex subset of $X$. It is a simple computation; we get $P_{C}(1,1,1)=(1,0,0), \Pi_{C}(1,1,1)=$ $(2,0,0)$.

We set $\rho=1$ is a positive number and define $f: C \rightarrow R$ by

$$
f(x)=-2-2 \sqrt{5}
$$

Then $f$ is convex and weakly continuous. Simple computations show that

$$
\Pi_{C}^{f}(1,1,1)=(4,0,0)
$$


Let $E$ be a Banach space, and let $C_{1}, C_{2}, C_{3}, \ldots$ be a sequence of weakly closed subsets of $E$. We denote by $s-L i_{n} C_{n}$ the set of limit points of $\left\{C_{n}\right\}$, that is, $x \in s-L i_{n} C_{n}$ if and only if there exists $\left\{x_{n}\right\} \subset E$ such that $\left\{x_{n}\right\}$ converges strongly to $x$ and that $x_{n} \in C_{n}$ for all $n \in N$. Similarly, we denote by $w-L s_{n} C_{n}$ the set of cluster points of $\left\{C_{n}\right\}, y \in w-L s_{n} C_{n}$ if and only if there exists $\left\{y_{n_{i}}\right\}$ such that $\left\{y_{n_{i}}\right\}$ converges weakly to $y$ and that $\left\{y_{n_{i}}\right\} \in C_{n_{i}}$ for all $i \in N$. Using these definitions, we define the Mosco convergence [2] of $C_{n_{i}}$. If $C_{0}$ satisfies

$$
s-L i_{n} C_{n}=C_{0}=w-L s_{n} C_{n}
$$

we say that $C_{n}$ is a Mosco convergent sequence to $C_{0}$ and write

$$
C_{0}=M-\lim _{n \rightarrow \infty} C_{n}
$$

Notice that the inclusion $s-L i_{n} C_{n} \subset w-L s_{n} C_{n}$ is always true. Therefore, in order to show the existence of $M-\lim _{n \rightarrow \infty} C_{n}$, it is sufficient to prove $w-L s_{n} C_{n} \subset s-L i_{n} C_{n}$. For more details, see [17].

\section{Main results}

\subsection{Generalized Mosco convergence theorems}

Theorem 3.1 Let $E$ be a smooth, reflexive, and strictly convex Banach space and $C$ be a nonempty closed convex subset of $E$. Let $C_{1}, C_{2}, C_{3}, \ldots$ be nonempty closed convex subsets of $C, f: E \rightarrow R$ be a convex and weakly continuous mapping with $C \subset \operatorname{int}(D(f))$. If $C_{0}=$ $M-\lim _{n \rightarrow \infty} C_{n}$ exists and is nonempty, then $C_{0}$ is a closed convex subset of $C$ and, for each $x \in C,\left\{\Pi_{C_{n}}^{f} x\right\}$ converges weakly to $\Pi_{C_{0}}^{f} x$.

Proof It is easy to prove that $C_{0}$ is closed and convex if $C_{n}$ is a closed convex subset of $C$ for each $n \in N$. Fix $x \in C$. For the sake of simplicity, we write $x_{n}$ instead of $\Pi_{C_{n}}^{f} x$ for $n \in N$. Since $C_{0}=M-\lim _{n \rightarrow \infty} C_{n}$, we have that for each $y \in C_{0}$, there exists $\left\{y_{n}\right\} \subset E$ such that $y_{n} \rightarrow y$ as $n \rightarrow \infty$ and that $y_{n} \in C_{n}$ for each $n \in N$. From Lemma 2.6, we have

$$
\left\langle x_{n}-y_{n}, J x-J x_{n}\right\rangle+\rho f\left(y_{n}\right)-\rho f(x) \geq 0 .
$$

Hence, we obtain

$$
\begin{aligned}
0 & \leq\left\langle x_{n}-x, J x-J x_{n}\right\rangle+\left\langle x-y_{n}, J x-J x_{n}\right\rangle+\rho f\left(y_{n}\right)-\rho f(x) \\
& \leq-\left(\|x\|-\left\|x_{n}\right\|\right)^{2}+\left(\|x\|+\left\|x_{n}\right\|\right)\left\|x-y_{n}\right\|+\rho f\left(y_{n}\right)-\rho f(x),
\end{aligned}
$$

thus,

$$
\left(\|x\|-\left\|x_{n}\right\|\right)^{2} \leq\left(\|x\|+\left\|x_{n}\right\|\right)\left\|x-y_{n}\right\|+\rho f\left(y_{n}\right)-\rho f(x) .
$$

Suppose that $\left\{x_{n}\right\}$ is not bounded. Then there exists a subsequence $\left\{x_{n_{i}}\right\}$ of $\left\{x_{n}\right\}$ such that $\left\|x_{n_{i}}\right\| \rightarrow \infty$. It follows that

$$
\frac{\|x\|^{2}}{\left\|x_{n_{i}}\right\|}-2\|x\|+\left\|x_{n_{i}}\right\| \leq\left(1+\frac{\|x\|}{\left\|x_{n_{i}}\right\|}\right)\left\|x-y_{n_{i}}\right\|+\frac{\rho f\left(y_{n_{i}}\right)-\rho f(x)}{\left\|x_{n_{i}}\right\|}
$$


for a sufficiently large number $i \in N$. As $i \rightarrow \infty$, we obtain $+\infty \leq\left\|x-y_{n_{i}}\right\|<+\infty$. This is a contradiction. Hence we have that $\left\{x_{n}\right\}$ is bounded.

Since $\left\{x_{n}\right\}$ is bounded, there exists a subsequence, again denoted by $\left\{x_{n}\right\}$, such that it converges weakly to $x_{0} \in C$. From the definition of $C_{0}$, we get $x_{0} \in C_{0}$.

Now, we prove that $\Pi_{C_{0}}^{f} x=x_{0}$. From weak lower semi-continuity of the norm and weak continuity of $f$, we have

$$
\begin{aligned}
\liminf _{n \rightarrow \infty} G\left(x_{n}, J x\right) & =\liminf _{n \rightarrow \infty}\left\|x_{n}\right\|^{2}-2\left\langle x_{n}, J x\right\rangle+\|x\|^{2}+\rho f\left(x_{n}\right) \\
& \geq\left\|x_{0}\right\|^{2}-2\left\langle x_{0}, J x\right\rangle+\|x\|^{2}+\rho f\left(x_{0}\right) \\
& =G\left(x_{0}, J x\right) .
\end{aligned}
$$

On the other hand, we get

$$
\begin{aligned}
\liminf _{n \rightarrow \infty} G\left(x_{n}, J x\right) & \leq \liminf _{n \rightarrow \infty} G\left(y_{n}, J x\right) \\
& =\liminf _{n \rightarrow \infty}\left\|y_{n}\right\|^{2}-2\left\langle y_{n}, J x\right\rangle+\|x\|^{2}+\rho f\left(y_{n}\right) \\
& =G(y, J x) .
\end{aligned}
$$

So,

$$
G\left(x_{0}, J x\right) \leq G(y, J x), \quad \forall y \in C_{0},
$$

that is,

$$
G\left(x_{0}, J x\right)=\inf _{y \in C_{0}} G(y, J x)
$$

Hence we get $\Pi_{C_{0}}^{f} x=x_{0}$.

According to our consideration above, each sequence $\left\{x_{n}\right\}$ has, in turn, a subsequence which converges weakly to the unique point $\Pi_{C_{0}}^{f} x$. Therefore, the sequence $\left\{x_{n}\right\}$ converges weakly to $\Pi_{C_{0}}^{f} x$. This completes the proof.

A Banach space $E$ is said to have the Kadec-Klee property if a sequence $\left\{x_{n}\right\}$ of $E$ satisfying that $x_{n} \rightarrow x_{0}$ and $\left\|x_{n}\right\| \rightarrow\left\|x_{0}\right\|$ converges strongly to $x_{0}$. It is known that $E^{*}$ has a Fréchet differentiable norm if and only if $E$ is reflexive, strictly convex, and has the KadecKlee property; see, for example, [10].

Theorem 3.2 Let E be a smooth Banach space such that $E^{*}$ has a Fréchet differentiable norm. Let $C$ be a nonempty closed convex subset of $E$. Let $C_{1}, C_{2}, C_{3}, \ldots$ be nonempty closed convex subsets of $C, f: E \rightarrow R$ be a convex and weakly continuous mapping with $C \subset \operatorname{int}(D(f))$. If $C_{0}=M-\lim _{n \rightarrow \infty} C_{n}$ exists and is nonempty, then $C_{0}$ is a closed convex subset of $C$ and, for each $x \in C,\left\{\Pi_{C_{n}}^{f} x\right\}$ converges strongly to $\Pi_{C_{0}}^{f} x$.

Proof Fix $x \in C$ arbitrarily. We write $x_{n}=\Pi_{C_{n}}^{f} x$ and $x_{0}=\Pi_{C_{0}}^{f} x$. By Theorem 3.1, we obtain $x_{n} \rightarrow x_{0}$. Since $E^{*}$ has a Fréchet differentiable norm, $E$ has the Kadec-Klee property. Therefore, it is sufficient to prove that $\left\|x_{n}\right\| \rightarrow\left\|x_{0}\right\|$ as $n \rightarrow \infty$. Since $x_{0} \in C_{0}$, there exists 
a sequence $\left\{y_{n}\right\} \subset C$ such that $y_{n} \rightarrow x_{0}$ as $n \rightarrow \infty$ and $y_{n} \in C_{n}$ for each $n \in N$. It follows that

$$
\begin{aligned}
G\left(x_{0}, J x\right) & \leq \liminf _{n \rightarrow \infty} G\left(x_{n}, J x\right) \\
& \leq \limsup _{n \rightarrow \infty} G\left(x_{n}, J x\right) \\
& \leq \limsup _{n \rightarrow \infty} G\left(y_{n}, J x\right) \\
& =G\left(x_{0}, J x\right) .
\end{aligned}
$$

Hence we obtain $G\left(x_{0}, J x\right)=\lim _{n \rightarrow \infty} G\left(x_{n}, J x\right)$. Since $\left\langle x_{n}, J(x)\right\rangle$ converges to $\left\langle x_{0}, J(x)\right\rangle$ and $f$ is weakly continuous, we get

$$
\lim _{n \rightarrow \infty}\left\|x_{n}\right\|=\left\|x_{0}\right\|
$$

Using the Kadec-Klee property of $E$, we obtain that $\left\{x_{n}\right\}$ converges strongly to $x_{0}$. This completes the proof.

Definition 3.3 ([18]) Let $C$ be a closed convex subset of a Banach space $E$, let $\left\{T_{n}\right\}_{n=1}^{\infty}$ be a countable family of mappings of $C$ into itself with the nonempty common fixed point set $F$. The $\left\{T_{n}\right\}_{n=1}^{\infty}$ is said to be uniformly closed if $x_{n} \rightarrow x$ and $\left\|x_{n}-T_{n} x_{n}\right\| \rightarrow 0$ as $n \rightarrow \infty$ implies $x \in F$.

\subsection{Strong convergence theorems}

Lemma 3.4 (see Lemma 3.3 of Klin-eam and Suantai [5]) Let $C$ be a closed convex subset of a Banach space $E$ and for all $n \in N$, let $\alpha=\left(\alpha_{1}, \alpha_{2}, \ldots, \alpha_{n}\right)$ such that $\alpha_{i} \geq 0, i=1,2, \ldots, n$, $\alpha_{1}>0$, and $\sum_{i=1}^{n} \alpha_{i}=1$. Let $T$ be an $\alpha$-nonexpansive mappingfrom $C$ into itself. If $\alpha_{1}>\frac{1}{\sqrt[n-1]{2}}$, let $\left\{x_{m}\right\}$ be a bounded sequence in $C$, then $\left\|x_{m}-T x_{m}\right\| \rightarrow 0$ if and only if $\left\|x_{m}-T_{\alpha} x_{m}\right\| \rightarrow 0$ as $m \rightarrow \infty$.

Lemma 3.5 ([6]) Let $C$ be a closed convex subset of a Banach space $E$, and for all $n \in N$, let $\alpha=\left(\alpha_{1}, \alpha_{2}, \ldots, \alpha_{n}\right)$ such that $\alpha_{i} \geq 0, i=1,2, \ldots, n, \alpha_{1}>0$, and $\sum_{i=1}^{n} \alpha_{i}=1$. Let $T$ be an $\alpha$-nonexpansive mapping from $C$ into itself. If $\alpha_{1}>\frac{1}{\sqrt[n-1 / \sqrt{2}]{2}}$, let $\left\{x_{m}\right\} \subset C$ converge strongly to $x$ and $\left\|x_{m}-T x_{m}\right\| \rightarrow 0$ converge strongly to 0 as $m \rightarrow \infty$, then $x \in F(T)$.

Lemma 3.6 ([6]) Let C be a closed convex subset of a uniformly convex and smooth Banach space $E$, and for all $n \in N$, let $\alpha=\left(\alpha_{1}, \alpha_{2}, \ldots, \alpha_{n}\right)$ such that $\alpha_{i} \geq 0, i=1,2, \ldots, n, \alpha_{1}>0$, and $\sum_{i=1}^{n} \alpha_{i}=1$. Let $T$ be an $\alpha$-nonexpansive mapping from $C$ into itself. If $\alpha_{1}>\frac{1}{\sqrt[n-1]{2}}$, then $F(T)$ is closed and convex.

Theorem 3.7 Let $C$ be a closed convex subset of a uniformly convex and smooth Banach space $E$, let $\left\{T_{n}\right\}_{n=1}^{\infty}$ be a uniformly closed countable family of $\alpha_{n}$-nonexpansive mappings of $C$ into itself such that $F:=\bigcap_{n=1}^{\infty} F\left(T_{n}\right) \neq \emptyset$, let $\alpha_{n}=\left(\alpha_{n 1}, \alpha_{n 2}, \ldots, \alpha_{n N_{0}}\right)$ such that $\alpha_{n i} \geq 0$, $i=1,2, \ldots, N_{0}, \alpha_{n 1}>0$, and $\sum_{i=1}^{N_{0}} \alpha_{n i}=1$. Let $f: E \rightarrow R$ be a convex and weakly continuous mapping with $C \subset \operatorname{int}(D(f))$. For any given Gauss $x_{0} \in E, C_{1}=C$, and $x_{1}=\Pi_{C_{1}}^{f} x_{0}$, define a 
sequence $\left\{x_{n}\right\}$ in $C$ by the following algorithm:

$$
\left\{\begin{array}{l}
y_{n}=\left(1-\beta_{n}\right) x_{n}+\beta_{n} T_{n} x_{n}, \\
C_{n+1}=\left\{z \in C_{n}:\left\|y_{n}-z\right\| \leq\left\|x_{n}-z\right\|\right\}, \\
x_{n+1}=\Pi_{C_{n+1}}^{f} x_{0}, \quad n \in N
\end{array}\right.
$$

where $0<a \leq \beta_{n} \leq 1$ for all $n \in N$. If $\alpha_{n 1}>\frac{1}{N_{0}-\sqrt[1]{2}}$, then $\left\{x_{n}\right\}$ converges strongly to $x^{*}=\Pi_{F}^{f} x_{0}$.

Proof Step 1. We show that $C_{n}$ is closed and convex for each $n \geq 0$.

From the definitions of $C_{n}$, it is obvious that $C_{n}$ is closed for each $n \geq 0$. Moreover, since $\left\|y_{n}-z\right\| \leq\left\|x_{n}-z\right\|$ is equivalent to

$$
\left\|y_{n}-x_{n}\right\|^{2}+2\left\langle y_{n}-x_{n}, J x_{n}-J z\right\rangle \leq 0
$$

so $C_{n}$ is convex for each $n \geq 0$.

Step 2. We show that $F \subset C_{n}$ for all $n \geq 0$. For all $p \in F$, we have that

$$
\begin{aligned}
\left\|y_{n}-p\right\|= & \left\|\left(1-\beta_{n}\right) x_{n}+\beta_{n} T_{n} x_{n}-p\right\| \\
\leq & \left(1-\beta_{n}\right)\left\|x_{n}-p\right\|+\beta_{n}\left\|T_{n} x_{n}-p\right\| \\
= & \left(1-\beta_{n}\right)\left\|x_{n}-p\right\|+\beta_{n} \| \alpha_{n 1}\left(T_{n} x_{n}-T_{n} p\right) \\
& +\alpha_{n 2}\left(T_{n} x_{n}-T_{n}^{2} p\right)+\cdots+\alpha_{n N_{0}}\left(T_{n} x_{n}-T_{n}^{N_{0}} p\right) \| \\
\leq & \left(1-\beta_{n}\right)\left\|x_{n}-p\right\|+\beta_{n} \frac{1-\alpha_{n 1}^{N_{0}-1}}{\alpha_{n 1}^{N_{0}-1}}\left\|x_{n}-T_{n} p\right\| \\
\leq & \left(1-\beta_{n}\right)\left\|x_{n}-p\right\|+\beta_{n}\left\|x_{n}-p\right\| \\
= & \left\|x_{n}-p\right\| .
\end{aligned}
$$

It implies that $p \in C_{n}$ for all $n \geq 0$. So, we have $F \subset C_{n}$ for all $n \geq 0$.

Step 3. We show that $\lim _{n \rightarrow \infty} x_{n}=x^{*}=\Pi_{\bar{C}^{\prime}}^{f} x_{0}$ and $x^{*} \in F$, where $\bar{C}=\bigcap_{n=1}^{\infty} C_{n}$. Indeed, since $\left\{C_{n}\right\}$ is a decreasing sequence of closed convex subsets of $E$ such that $\bar{C}=\bigcap_{n=1}^{\infty} C_{n}$ is nonempty, it follows that

$$
M-\lim _{n \rightarrow \infty} C_{n}=\bar{C}=\bigcap_{n=1}^{\infty} C_{n} \neq \emptyset .
$$

By Theorem 3.2, we get

$$
x_{n} \rightarrow x^{*} \quad \text { as } n \rightarrow \infty \text {. }
$$

Noticing that $x_{n+1}=\Pi_{C_{n+1}}^{f} x_{0} \in C_{n+1}$, we obtain that

$$
\left\|y_{n}-x_{n+1}\right\| \leq\left\|x_{n}-x_{n+1}\right\|
$$

In view of (3.2), we have that

$$
\left\|y_{n}-x_{n+1}\right\| \rightarrow 0 \quad \text { as } n \rightarrow \infty
$$


and

$$
\left\|y_{n}-x_{n}\right\| \leq\left\|y_{n}-x_{n+1}\right\|+\left\|x_{n+1}-x_{n}\right\| \rightarrow 0 \quad \text { as } n \rightarrow \infty \text {. }
$$

From $y_{n}=\left(1-\beta_{n}\right) x_{n}+\beta_{n} T_{n} x_{n}$, we have

$$
\left\|x_{n}-T_{n} x_{n}\right\|=\frac{1}{\beta_{n}}\left\|y_{n}-x_{n}\right\| .
$$

Because of the assumption that $0<a \leq \beta_{n} \leq 1$, we have

$$
\lim _{n \rightarrow \infty}\left\|x_{n}-T_{n} x_{n}\right\|=0
$$

Since $\left\{x_{n}\right\}$ is uniformly closed, then $x^{*} \in F$.

Step 4. We show that $x^{*}=\Pi_{F}^{f} x_{0}$. Since $x^{*}=\Pi_{\bar{C}}^{f} x_{0} \in F$ and $F$ is a nonempty closed convex subset of $\bar{C}=\bigcap_{n=1}^{\infty} C_{n}$, we conclude that $x^{*}=\Pi_{F}^{f} x_{0}$. This completes the proof.

Corollary 3.8 ([6]) Let $C$ be a closed convex subset of a uniformly convex and smooth Banach space $E$, let $T$ be an $\alpha$-nonexpansive mapping of $C$ into itself such that $F(T) \neq \emptyset$, let $\alpha=\left(\alpha_{1}, \alpha_{2}, \ldots, \alpha_{N_{0}}\right)$ such that $\alpha_{i} \geq 0, i=1,2, \ldots, N_{0}, \alpha_{1}>0$, and $\sum_{i=1}^{N_{0}} \alpha_{i}=1$. For any given Gauss $x_{0} \in E, C_{1}=C$, and $x_{1}=\Pi_{C_{1}} x_{0}$, define a sequence $\left\{x_{n}\right\}$ in $C$ by the following algorithm:

$$
\left\{\begin{array}{l}
y_{n}=\left(1-\beta_{n}\right) x_{n}+\beta_{n} T x_{n}, \\
C_{n+1}=\left\{z \in C_{n}:\left\|y_{n}-z\right\| \leq\left\|x_{n}-z\right\|\right\}, \\
x_{n+1}=\Pi_{C_{n+1}} x_{0}, \quad n \in N,
\end{array}\right.
$$

where $0<a \leq \beta_{n} \leq 1$ for all $n \in N$. If $\alpha_{1}>\frac{1}{N_{0}-1} \sqrt{2}$, then $\left\{x_{n}\right\}$ converges strongly to $x^{*}=\Pi_{F} x_{0}$.

Proof Substituting $T$ to $T_{n}$ in the proof of Theorem 3.7 and putting $f(x) \equiv 0$, we can draw from Theorem 3.7 the desired conclusion immediately.

Remark 3.9 Theorem 3.7 extends the main results of [6] from a single mapping to a countable family of mappings and from the generalized projection operator to the modified generalized $f$-projection operator by a new method.

Competing interests

The authors declare that they have no competing interests.

Authors' contributions

All authors contributed equally and significantly in writing this article. All authors read and approved the final manuscript. 


\section{References}

1. Chidume, C: Geometric Properties of Banach Spaces and Nonlinear Iterations. Lecture Notes in Mathematics, vol. 1965. Springer, London (2009)

2. Wittmann, R: Approximation of fixed points of nonexpansive mappings. Arch. Math. 58, 486-491 (1992)

3. Berinde, V: Iterative Approximation of Fixed Points, 2nd edn. Lecture Notes in Mathematics, vol. 1912. Springer, Berlin (2007)

4. Goebel, K, Pineda, MA: On a type of generalized nonexpansiveness. In: Proc. of the 8th International Conference on Fixed Point Theory and Its Application, vol. 74, pp. 660-665 (2007)

5. Klin-eam, C, Suantai, S: Fixed point theorems for $\alpha$-nonexpansive mappings. Appl. Math. Lett. 23, 728-731 (2010)

6. Wang, Z, Su, Y, Kang, J: Hybrid algorithm for an $\alpha$-nonexpansive mappings in a Banach space. J. Nonlinear Sci. Appl. 5(1), 56-63 (2012)

7. Alber, Y: Generalized projection operators in Banach spaces: properties and applications. In: Proceedings of the Israel Seminar, Ariel. Israel Funct. Differ. Equ., vol. 1, pp. 1-21 (1994)

8. Alber, Y: Metric and generalized projection operators in Banach spaces: properties and applications. In: Kartsatos, A (ed.) Theory and Applications of Nonlinear Operators of Accretive and Monotone Type, pp. 15-50. Dekker, New York (1996)

9. Li, JL: The generalized projection operator on reflexive Banach spaces and its applications. J. Math. Anal. Appl. 306, 55-71 (2005). doi:10.1016/j.jmaa.2004.11.007

10. $\mathrm{Wu}, \mathrm{KQ}$, Huang, NJ: The generalized $f$-projection operator with an application. Bull. Aust. Math. Soc. 73, 307-317 (2006). doi:10.1017/S0004972700038892

11. Fan, JH, Liu, X, Li, JL: Iterative schemes for approximating solutions of generalized variational inequalities in Banach spaces. Nonlinear Anal. TMA 70, 3997-4007 (2009). doi:10.1016/j.na.2008.08.008

12. Cioranescu, I: Geometry of Banach Spaces, Duality Mappings and Nonlinear Problems. Kluwer Academic, Dordrecht (1990)

13. Takahashi, W: Nonlinear Functional Analysis. Yokohama Publishers, Yokohama (2000)

14. Vainberg, MM: Variational Methods and Method of Monotone Operators. Wiley, New York (1973)

15. Li, X, Huang, N, O'Regan, D: Strong convergence theorems for relatively nonexpansive mappings in Banach spaces with applications. Comput. Math. Appl. 60, 1322-1331 (2010)

16. Alber, Y, Butnariu, D: Convergence of Bregman projection methods for solving consistent convex feasibility problems in reflexive Banach spaces. J. Optim. Theory Appl. 92, 33-61 (1997). doi:10.1023/A:1022631928592

17. Beer, G: Topologies on Closed and Closed Convex Sets. Mathematics and Its Applications, vol. 268. Kluwer Academic, Dordrecht (1993)

18. Zhang, J, Su, Y, Cheng, Q: Uniformly closed replaced AKTT or *AKTT condition to get strong convergence theorems for a countable family of relatively quasi-nonexpansive mappings and systems of equilibrium problems. Fixed Point Theory Appl. 2014, 103 (2014)

10.1186/1029-242X-2014-305

Cite this article as: Cheng et al.: Convergence theorems for modified generalized $f$-projections and generalized nonexpansive mappings. Journal of Inequalities and Applications 2014, 2014:305

\section{Submit your manuscript to a SpringerOpen ${ }^{\circ}$ journal and benefit from:}

- Convenient online submission

- Rigorous peer review

- Immediate publication on acceptance

- Open access: articles freely available online

- High visibility within the field

- Retaining the copyright to your article 